\title{
PREECLAMPSIA LEVE: CUIDADOS EN CASA
}

\author{
Margarita Pérez* , Olga Lucía Prieto**, Christi Dianne Barrera Triviño***, Mónica Patricia Benavides Ariza***, \\ Liliana Bernal Duque ${ }^{* * *}$, Diana Carolina Franco Susatama***, Marcela Sánchez Orozco***
}

\section{Resumen}

El presente estudio tiene como objetivo detectar las necesidades de cuidado percibidas por gestantes con diagnóstico de preeclampsia leve que manejan su patología en casa. Se realizó una investigación cualitativa descriptiva exploratoria entrevistando mujeres gestantes con este diagnóstico atendidas en el Hospital de San José de Bogotá DC. Para determinar el número que se necesitaba se tuvo en cuenta el principio de saturación, el cual se cumplió con ocho pacientes. El análisis de los datos fue hecho por edición y fundamentado en la teoría de suplencia o ayuda a través del modelo de necesidades humanas para la vida y la salud, como núcleo en la acción de enfermería de Virginia Henderson, en el cual se encontró que estas enfermas requieren un estricto cuidado en el manejo de cuatro necesidades básicas que son fisiológicas, de seguridad, de amor y pertenencia, y de autorrealización. En cada una de ellas las más relevantes fueron: toma de tensión arterial, tranquilidad, afecto de su familia y más información acerca de la patología que padecen. La realidad vista en la presente investigación genera gran expectativa frente a la importancia de la participación del enfermero profesional en el cuidado de la gestante con la patología mencionada, tanto en el área de promoción y prevención como en el diagnóstico precoz, tratamiento oportuno y limitación del daño.

Palabras clave: preecplampsia, cuidado, Virginia Henderson.

Abreviaturas: PRE-L, preeclampsia leve.

\section{HOME CARE IN MILD PRE - ECLAMPSIA}

\section{Abstract}

The purpose of this study is to detect the perceptions on required care of women with mild pre-eclampsia who handle their condition at home. A qualitative, descriptive, exploratory research was undertaken surveying women with this diagnosis seen at the San José Hospital in Bogotá D.C. The saturation principle was used to determine the required number, which was met by eight patients. Data analysis was performed by edition and was based on the substitution theory or help through human necessities for life and health, as the fundamentals of the Virginia Henderson theories, which found that this condition requires strict management of four essential necessities, that is, physiological needs, love assurance, emotional bonds (attachment) and self-realization. The most relevant in each of them were: taking of blood pressure, tranquility, receiving love and reassurance from their families and being more informed about their condition. The actual results of this research cause great expectations as to the importance of the nursing professional in the care of these patients, especially in the promotion of health and prevention of disease as well as on timely diagnosis and treatment and limitation of harm.

Key words: pre-eclampsia, care, Virginia Henderson.

Fecha recibido: noviembre 14 de 2008 - Fecha aceptado: mayo 8 de 2009

* Enfermera especialista en UCl. Jefe del Depto. de Enfermería del Hospital de San José. Profesora Asociada de cuidado crítico de la Facultad de Enfermería. Fundación Universitaria de Ciencias de la Salud. Bogotá DC. Colombia.

** Especialista en docencia universitaria. Profesora Asociada de Cuidado del adulto de la Facultad de Enfermería de la Fundación Universitaria de Ciencias de la Salud, Bogotá DC. Colombia.

*** Estudiantes de pregrado Facultad de Enfermería, Fundación Universitaria de Ciencias de la Salud, Bogotá DC. Colombia.

Merideidy Plazas; Asistente Metodológica. M. P. Epidemióloga. Docente División de Investigaciones, Fundación Universitaria de Ciencias de la Salud. Bogotá DC, Colombia. 


\section{Introducción}

Cualquier mujer en algún instante de su vida piensa sobre lo hermoso y gratificante que puede ser experimentar el fenómeno de la maternidad, siendo un milagro único y exclusivo hecho vida en el vientre materno. Sin embargo, no hay duda que también es una experiencia que impacta y se vive de manera diferente según la situación; por tanto, puede ser positiva o negativa para cada mujer. Existen situaciones o condiciones patológicas que imponen diferencia y convierten la gestación en un embarazo de alto riesgo, en el cual el objetivo principal del personal de salud implicado en el cuidado de la paciente es prevenir complicaciones irreversibles para el binomio, madre e hijo, entre las cuales la más grave es la muerte de alguno de los dos.

Para comprender la situación y analizarla desde un terreno cercano a la realidad es preciso mencionar que "en Bogotá la causa de mortalidad observada en 1998 es atribuida en el $28,8 \%$ a preeclampsia y eclampsia (toxemia)." El evento más frecuente (47\%) asociado con la presencia de preeclampsia es la hipertensión con proteinuria inducida por el embarazo. ${ }^{1}$ Además, "las causas básicas de muerte materna registradas durante el 2004 fueron los trastornos hipertensivos asociados con embarazo (eclampsia, preeclampsia e hipertensión inducida por el embarazo) y las hemorragias en el posparto, uno de cuyos factores de riesgo de muerte materna fue la inasistencia de las gestantes al control prenatal." ${ }^{.2} \mathrm{La}$ función primordial del equipo de salud y en especial del enfermero profesional es prevenir las posibles complicaciones de la gestación o bien que al presentarse éstas sean detectadas en su fase inicial para iniciar el tratamiento oportuno, lo cual se logra mediante la educación sobre los cuidados que debe tener la gestante en casa y con ello obtener madres y productos más sanos y disminuir las tasas de morbimortalidad maternoperinatal. ${ }^{3}$

Es así como en el presente estudio se destaca la importancia de la labor de enfermería, que radica en conocer las necesidades de cuidado de gestantes con PRE-L que son de manejo ambulatorio, en quienes se hace necesaria la educación sobre los cuidados en casa, teniendo en cuenta que son ellas mismas las encargadas de satisfacer sus necesidades tanto físicas como emocionales. $\mathrm{La}$ información brindada a la población objeto de estudio beneficiará a la gestante, su hijo, la familia y también al personal de salud para orientar el manejo ambulatorio, aportando parámetros que permitan controlar su patología de manera adecuada en su hogar.

Para lograr este objetivo el presente trabajo está soportado en la teoría de suplencia o ayuda de Virginia Henderson (1966), que se fundamenta en el cuidado manifestado a través del modelo de necesidades humanas para la vida y la salud como núcleo en la acción de enfermería, quien a su vez concibe el rol del enfermero como productor y realizador de acciones que en algún momento de su vida el paciente no puede hacer. Más que un modelo, su obra se considera como una filosofía de la profesión de enfermería, que define la función como la atención a la persona sana o enferma en todo tipo de actividades que contribuyan a mantener la salud o recuperarla, buscando conseguir un ser humano independiente lo antes posible para cubrir sus necesidades básicas, todo esto mediante un plan de cuidados elaborado de acuerdo con las necesidades detectadas. La autora parte del principio de que todos los seres humanos tienen una serie de necesidades básicas que deben satisfacer, independiente de la situación en que se encuentren y que puede variar el modo de satisfacerlas por cuestiones culturales, modos de vida o motivaciones, entre otros.

Estas consideraciones fundamentan la inclusión de las catorce necesidades básicas del paciente propuestas por Virginia Henderson:

- Respirar de manera normal.

- Comer y beber en forma adecuada.

- Eliminar residuos corporales.

- Moverse y mantener una postura conveniente.

- Dormir y descansar.

- Seleccionar, ponerse y quitarse la ropa adecuada.

- Mantener la temperatura corporal, controlando la ropa y el ambiente.

- Mantener limpieza e integridad en la piel. 
- Evitar los peligros ambientales e impedir que perjudiquen a otros.

- Comunicarse para expresar emociones, necesidades, temores, etc.

\section{- Profesar su religión.}

- Trabajar en alguna actividad que produzca una sensación de rendir provecho.

- Jugar o participar en diversas actividades recreativas.

- Aprender, descubrir o satisfacer la curiosidad que conduce al desarrollo normal y a la salud, y hacer uso de las instalaciones sanitarias disponibles.

De esta manera "las embarazadas críticamente enfermas representan un reto para el equipo de salud debido a los cambios fisiológicos propios de este estado, ya que se trata de dos pacientes (madre y feto) con fisiologías y necesidades distintas, cambios anatómicos en la madre y, por último, a la presencia de patologías exclusivas del embarazo como la preeclampsia". ${ }^{3}$ La mujer con esta patología puede sufrir complicaciones severas, tanto durante la gestación como al momento del parto o luego del nacimiento del niño. Por esto se hace necesario indagar sobre sus necesidades para contribuir a prevenirlas.

Este trabajo es de tipo cualitativo descriptivo exploratorio, incluyó gestantes que acudieron a la consulta de ginecoobstetricia del Hospital de San José de octubre a diciembre de 2005, diagnosticadas con PRE$\mathrm{L}$ por el especialista encargado del servicio de alto riesgo materno, con tratamiento individual según protocolo establecido en el hospital. Para decidir cuántas pacientes se necesitaban para este estudio, se tuvo en cuenta el principio de saturación teórica, individual y colectiva, y se alcanzó con ocho pacientes. La recolección de los datos se realizó en dos entrevistas semiestructuradas; en la de primer contacto se le informó la existencia del estudio de investigación y se le propuso participar en el mismo de manera voluntaria, por medio del consentimiento informado que concede el permiso a los investigadores de continuar con la entrevista de segundo contacto, que fue registrada en medio magnético y coordi- nada por una de las asesoras temáticas, en compañía de una de las estudiantes de octavo semestre de la Facultad de Enfermería.

Se realizó una prueba piloto con el fin de determinar el manejo de las preguntas en la entrevista, la claridad y la estructura de la misma, así como también establecer la facilidad de la recolección de los datos de manera escrita. Se aplicó en un día de consulta a dos de las pacientes que cumplían con los criterios de inclusión. El análisis de datos se realizó por edición, consistente en que el grupo investigador hizo las veces de intérprete que lee y analiza la información recogida para detectar semejanzas y clasificar la información. ${ }^{4}$ Luego se desarrolló un esquema de categorización para organizar los datos y a continuación se buscaron los patrones que conectaban las categorías. ${ }^{4}$ En el presente trabajo se mantienen los principios de beneficencia y respeto a la dignidad humana, como parte de derecho de autodeterminación después de brindar una información consistente acerca del propósito del estudio y los parámetros que se utilizarían. ${ }^{5} \mathrm{El}$ estudio no compromete la naturaleza moral ni ética de la población a estudiar, debido a que no hubo intervención por parte del grupo investigador.

Se realizaron ocho entrevistas grabadas con las cuales se completó el principio de saturación. Las entrevistadas manifestaron las siguientes necesidades que fueron organizadas a partir de las respuestas de acuerdo con la categorización de las necesidades de la teoría de Virginia Henderson (Tabla 1). Se puede observar que en las necesidades fisiológicas todas manifestaron necesitar control de la tensión arterial. En las necesidades de seguridad, es llamativo el bajo porcentaje que consideraron necesario el uso de medicamentos, en contraste con la totalidad que requirió tranquilidad. El apoyo afectivo fue importante en especial cuando procede de su núcleo familiar. Se encontró un alto índice de desconocimiento sobre su patología, así como también la abstinencia de responder atribuida tal vez al primero.

El presente trabajo está soportado en la teoría de suplencia o ayuda representada en Virginia Henderson (1966), que se fundamenta en el cuidado manifestado a través del modelo de necesidades humanas para la vida y la salud como núcleo en la acción de enfermería. ${ }^{6}$ La mayor parte de su teoría está basada como se define en su 
Tabla I. Categorización de necesidades según la clasificación de Virginia Henderson

Necesidades fisiológicas: respirar de forma normal, comer y beber de manera adecuada, eliminar residuos corporales, dormir y descansar, mantener la temperatura corporal controlando la ropa y el ambiente.

Necesidades de seguridad: mantener limpieza e integridad
en la piel, evitar los peligros ambientales e impedir que
perjudiquen a otros.

Necesidad de amor y pertenencia: comunicarse con otros para expresar emociones, necesidades, temores, etc. Profesar su religión.

Necesidad de autorrealización: trabajar en alguna actividad que produzca una sensación de rendir provecho, jugar o participar en diversas actividades recreativas, aprender, descubrir o satisfacer la curiosidad que conduce al desarrollo normal y a la salud, $y$ hacer uso de las instalaciones sanitarias disponibles.

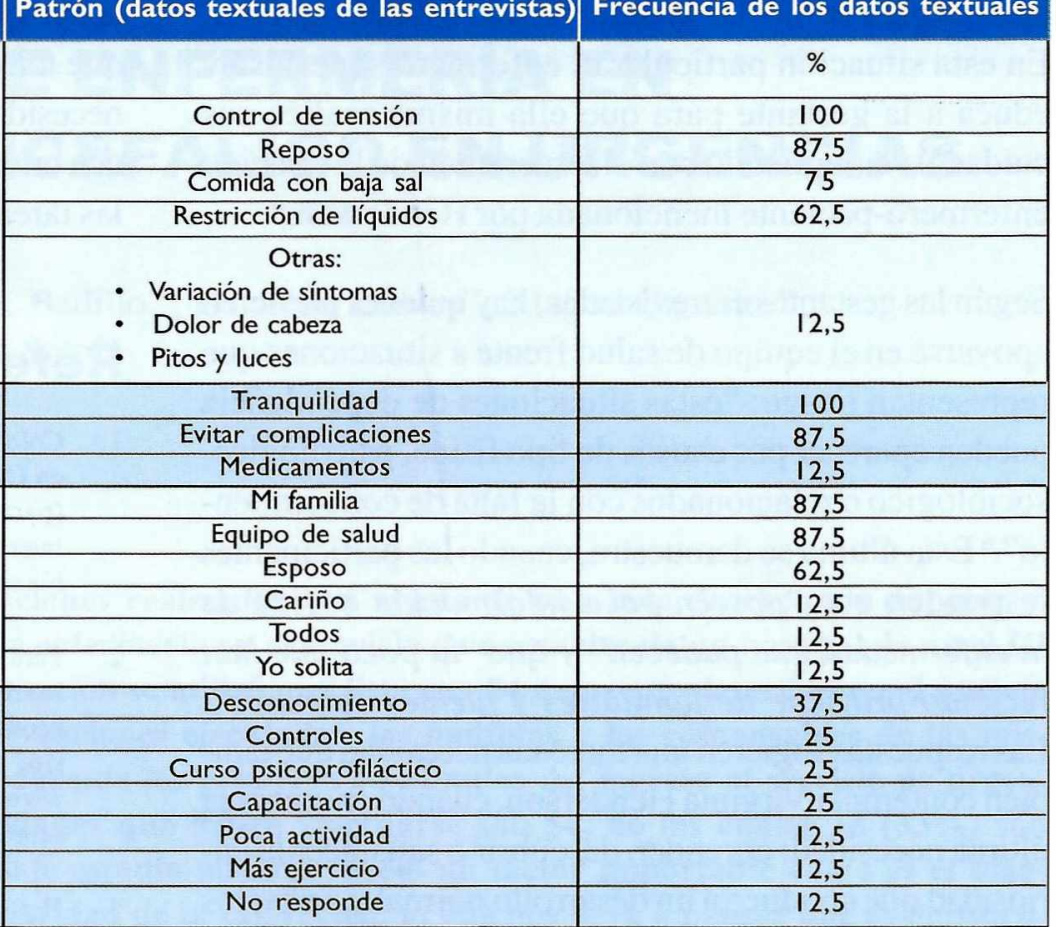

libro The Nature of Nursing, en la fisiología, medicina, psicología y física. También utiliza la observación de su práctica diaria para dar forma a su teoría ${ }^{6}$ y define la necesidad fundamental como "todo aquello que es esencial al ser humano para mantener su vida o asegurar su bienestar, siendo concebida esta necesidad como requisito más que como una carencia". ${ }^{6}$ Por lo tanto, una vez realizadas las entrevistas fueron analizadas basándonos en esta teoría y empleando el método por edición. Los resultados encontrados indican que la tranquilidad y el reposo son fundamentales para la recuperación de las pacientes y que está de acuerdo con lo descrito en la literatura mundial en especial con el trabajo de Virginia Henderson, quien los referenció como necesidades fisiológicas.

La mayoría de las pacientes del presente estudio presentaron temor cuando fueron diagnosticadas con PREL, situación que se alcanza a considerar normal debido a que en cualquier circunstancia una persona se puede alterar al enterarse que padece algún tipo de enfermedad que requiere cuidado especializado. Ante esta situación la necesidad que se manifiesta es la de seguridad según Virginia Henderson, cuya solución es brin- darle a la paciente un ambiente de tranquilidad para manejar su patología, teniendo en cuenta que día tras día las pacientes requieren cuidado y aún más cuando saben que tienen PRE-L, debido a que después de ser informadas sobre la sintomatología y posibles complicaciones, deben asumir con mayor responsabilidad el cuidado de la enfermedad; es entonces cuando recobra el sentido la función del enfermero según Henderson, ${ }^{6}$ cuando se debe atender a la persona sana o enferma en todo tipo de actividades que contribuyan a mantener la salud o a recuperarla, como por ejemplo, promover el control constante de la tensión, la puntualidad en las citas médicas, la realización de exámenes de sangre y uno de los cuidados más importantes que es la buena alimentación, tal y como diría Virginia Henderson el comer y beber de manera adecuada son necesidades fisiológicas que proporcionan un cambio en el organismo. Esto lo demuestran las gestantes durante la entrevista; empíricamente o no, ellas actúan de acuerdo con lo mencionado en la bibliografía revisada durante el proceso de investigación. Jaramillo ${ }^{7}$ por ejemplo, afirma que en relación con el tratamiento, se hace necesario implementar cuidados en la alimentación como 
el aumento moderado en el consumo de proteínas y el reposo sobre uno de los costados, que pueden ser suficientes para controlar la enfermedad estando en casa. En esta situación particular el enfermero supervisa y educa a la gestante para que ella misma realice sus cuidados, cumpliendo con el tercer nivel de la relación enfermero-paciente mencionada por Henderson. ${ }^{6}$

Según las gestantes entrevistadas, hay quienes prefieren apoyarse en el equipo de salud frente a situaciones que representan riesgo: "estas situaciones de dependencia pueden aparecer por causas de tipo físico, psicológico, sociológico o relacionados con la falta de conocimiento". ${ }^{6}$ Esta última se demuestra, cuando las participantes responden que "poseen poca información acerca de la enfermedad que padecen" y que "la poca que han recibido proviene de familiares y fuentes cercanas". Las respuestas sugieren una marcada necesidad que también contempla Virginia Henderson, cuando enumera la última necesidad: aprender, descubrir o satisfacer la curiosidad que conduce a un desarrollo normal y a usar los recursos disponibles.

Observando las anteriores situaciones se encuentra una marcada necesidad del enfermero como acompañante, ${ }^{6}$ pues será quien busca identificar e interpretar las necesidades de cuidado de la población como es aprender, descubrir o satisfacer la curiosidad que conduce a adquirir el conocimiento que permita el desarrollo normal y el mantenimiento de la salud, para llegar a feliz término el embarazo. Por ello, el personal profesional preparado y con conocimientos propios de la enfermedad, es quien puede proporcionar un ambiente favorable para estas pacientes, jugando un papel trascendental junto con los familiares y el equipo de salud para encontrar soluciones y prácticas seguras al enfrentar la enfermedad; pero esto no es una tarea fácil sin contar con un buen grupo de apoyo que incluya al esposo, los parientes, amigos y otros que también ayuden a la gestante enferma a suplir las necesidades de amor y pertenencia. Estas personas pueden brindarles información, apoyo emocional o ayuda con las tareas del hogar.

\section{Referencias}

1. Colombia. Ministerio de Salud. Panorama de la mortalidad materna en Colombia, 1995 - 2000: logros, fracasos, compromisos y retos (parte II). SIVIGILA: Informe Ejecutivo Semanal [serie en Internet]. 2001 sep. [citado 1 Jul 2009]; 38:[aprox. 3 p.]. Disponible en: http://www.col.ops-oms.org/sivigila/2001/bole38_2001.htm

2. Perú. Ministerio de Salud. Dirección General de Salud de las Personas. Plan nacional para la reducción de la muerte materna, fetal y neonatal 2004-2006 [monografía en Internet]. Lima: El Ministerio; 2004 [citado 1 Jul. 2009]. Disponible en: http:// www.minsa.gob.pe/otraspublicaciones/29/Plan\%20Reduccion $\% 20$ Mortalidad\%20MFN\%201-MCC.doc

3. Herrera J. Evaluación del riesgo obstétrico en el cuidado prenatal. Colomb Med [serie en Internet]. 2002 [citado 1 Jul 2009]; 333 (1): [aprox. 4 p.]. Disponible en: http://www.bioline.org.br/ request?rc02004

4. Hungler P, Polit D. Investigación científica en ciencias de la salud. 6a ed. México: McGraw-Hill Interamericana; 2000.

5. Colombia. Ministerio de Salud. Resolución 8430 de 1993, por la cual se establecen las normas científicas, técnicas y administrativas para la investigación en salud.

6. Tendencias y modelos más utilizados en el cuidado de enfermería. [monografía en Internet]. Almería, España: Antonio José Ibarra Fernández; 2007[citado 1 Jul 2009]. Disponible en: http:// www.aibarra.org/Apuntes/Fundamentos/default.htm

7. Jaramillo N. Pre-eclampsia y eclampsia [monografía en Internet]. Cartagena : Contusalud; 2001 [citado 1 Jul. 2009]. Disponible en: http://www.contusalud.com/sepa_embarazo_preclampsia.htm 